\title{
A Simple Continuum Model for Simulation of Boron Interstitial Clusters based on Atomistic Calculations
}

\author{
Srinivasan Chakravarthi ${ }^{a}$ and Scott T. Dunham ${ }^{b}$ \\ ${ }^{a}$ Department of Manufacturing Engineering \\ ${ }^{6}$ Department of Electrical and Computer Engineering \\ Boston University, Boston, MA 02215, USA.
}

\begin{abstract}
Boron exhibits anomalous diffusion during the initial phases of ion implant annealing. Boron TED is characterized by enhanced tail diffusion coupled with an electrically inactive immobile peak. The immobile peak is due to clustering of boron in the presence of excess interstitials which also cnhance boron diffusion in the tail region. In this paper we present a simple model for the formation of immobile boron clusters and associated point defect interactions derived based on atomistic calculations.
\end{abstract}

\section{Introduction}

Ion implantation is the primary means of dopant introduction in VLSI fabrication. It is well known that implantation introduces damage that on annealing leads to the phenomenon of transient enhanced diffusion (TED). Implantation introduces a large number of point defects orders of magnitude higher than the dopant concentration. These excess interstitials and vacancies recombine with each other during the initial stages of annealing. The remaining excess point defects also form extended defects, primarily $\{311\}$ defects for interstitials and boron interstitial clusters (BICs) for boron [1]. These defects interact with each other through changes in point defect and solute concentrations.

Under TED conditions, boron is found to be immobilized at concentrations well below solid solubility. This has been explained on the basis of the formation of boron interstitial clusters (BICs). Models using either a moment-based approach [2] or a discrete set of cluster compositions $[3,4]$ have been successfully used for modeling of boron interstitial clusters. A problem with both of these approaches is that they lead to complicated models with associated long simulation times and large sets of non-unique parameters.

In this paper we derive a simple single cluster model for BICs derived from a multicluster model based on ab-initio calculations performed at Lawrence Livermore National Labs [5]. Despite its simplicity, the model accurately describes boron clustering and anomalous diffusion over a wide range of experimental conditions. The resulting model can be easily incorporated into an industrial process simulator. 


\section{Multi-Cluster Models for Boron TED}

Boron aggregation is a complicated process as there is a luge array of potential cluster compositions. In previous work, Lilak et al. [3] presented a boron clustering model based on the same calculations which we use in this work. Pelaz et al. [4] derived a similar model, but with a somewhat different parameter set. In both cases, they considered a large range of clusters as shown in Fig. 1(a), with an associated large set of continuity equations and parameters.

The model implemented in this work uses kinetic reactions that lead to the formation of clusters. For example, a substitutional boron can react with a silicon self interstitial to form an immobile BI which can further react with another interstitial to form a $\mathrm{BI}_{2}$ cluster or with an interstitial boron $\left(\mathrm{B}_{i}\right)$ to give $\mathrm{B}_{2} \mathrm{I}_{2}$. For reactions of the form $A+B \Leftrightarrow C$, the rate of formation of $C$ is given by,

$$
R=4 \pi r_{\text {cap }}\left(D_{\mathrm{A}}+D_{\mathrm{B}}\right)\left(C_{\mathrm{A}} C_{\mathrm{B}}-\frac{C_{\mathrm{C}}}{K_{\mathrm{eq}}}\right)
$$

where $r_{\text {cap }}$ is the capture radius of the reaction and $D$ and $C$ represent diffusivity and concentration. Cluster energetics calculations from $\mathrm{Zhu}$ et al. [5] were used as the basis for the simulations, with 10 different clusters considered: $\mathrm{BI}, \mathrm{BI}_{2}, \mathrm{~B}_{2} \mathrm{I}, \mathrm{B}_{2} \mathrm{I}_{2}$, $\mathrm{B}_{3} \mathrm{I}, \mathrm{B}_{3} \mathrm{I}_{2}, \mathrm{~B}_{4} \mathrm{I}_{2}, \mathrm{~B}_{4} \mathrm{I}, \mathrm{B}_{2}, \mathrm{~B}_{3}$.

Our analysis of this system found that most of the clusters rapidly achicve dynamic equilibrium with the free boron and interstitial concentrations, suggesting the possibility of reducing the number of equations and parameters needed to describe the system. As shown in Fig. 1(b), except for $B_{3} I$ and $B_{4} I_{2}$, all the clusters reach dynamic equilibrium with the $B$ and I concentration within a very short time, while the two larger clusters are also in local dynamic equilibrium. At the same time, only a small subset of the clusters are ever present in significant numbers. From Fig. 2(a) we can see that $\mathrm{BI}_{2}$ are the dominant cluster at very short times when the interstitial supersaturation is large. (Note that at such short times the $B_{3} I$ and $B_{4} I_{2}$ clusters are negligible due to the slow formation of $B_{3} I$.) Once $\{311\}$ defects form, the interstitial concentration drops, and as can be seen from Fig. $2(b)$, the dominant species is $B_{3} I$.

\section{Single-Cluster Model}

Based on the above observations, we can simplify the system of immobile clusters from ten to just one continuity equation, that for $B_{3} I$, with the rate of formation given by

$$
R_{\mathrm{B}_{3} \mathrm{I}}=k_{\mathrm{B}_{3} \mathrm{I}}^{r}\left(K_{\mathrm{B}_{3} \mathrm{I}} \mathrm{C}_{\mathrm{B}}^{3} \mathrm{C}_{\mathrm{I}}-\mathrm{C}_{\mathrm{B}_{3} \mathrm{I}}\right)
$$

In addition, we can also ignore the concentrations of small clusters other than $\mathrm{BI}_{2}$, which we express in terms of the free boron and interstitial concentrations:

$$
C_{B I_{2}}=K_{B I_{2}} C_{B} C_{I}^{2}
$$

We compared our simplified model to the full system and found that the results are virtually indistinguishable. A moment-based model characterized based on TEM results was used for $\{311\}$ defect kinetics [2]. Figs. 3(a) and (b) show examples of this comparison as well as to data from Intel [6] for TED at $800^{\circ} \mathrm{C}$. Similar agreement was obtained at higher and lower temperatures $\left(700\right.$ and $\left.900^{\circ} \mathrm{C}\right)$ as well as for other implant doses. 


\section{Conclusions}

In summary, we found that it is possible to develop a very simple model which accurately accounts for boron TED and clustering behavior. Based on analysis of cluster kinetics and energetics resulting from $a b$-initio calculations, we are able to match the results of a full multi-cluster model, while reducing the number of cluster continuity equations from ten to just one and the number of parameters from over twenty to only three. The resulting model clearly illuminates the critical processes involved in boron clustering.

\section{References}

[1] D.J. Eaglesham, P.A. Stolk, H.J. Gossmann, T.E. Haynes, and J.M. Poate, "Implant damage and transient enhanced diffusion in Si," Nuc. Inst. and Meth. B, vol. 106, p. 191, 1995.

[2] A.H. Gencer, S. Chakravarthi, and S.T. Dunham, "Physical modeling of transient enhanced diffusion and dopant deactivation via extended defect evolution," SISPAD '97 Proceedings, p. 77, 1997.

[3] A.D. Lilak, S.K. Earles, K.S. Jones, M.E. Law, and M.D. Giles, "A physics-based modeling approach for the simulation of anamalous boron diffusion and clustering behaviors," IEDM Technical Digest, p. 493, 1997.

[4] L. Pelaz, M. Jaraiz, G. H. Gilmer, H. J. Gossmann, C. S. Rafferty, and D. J. Eaglesham, "B diffusion and clustering in ion implanted Si: The role of B cluster precursors," Appl. Phys. Lett., vol. 70, no. 17, p. 2285, 1991.

[5] J. Zhu, M.-J. Caturla, M. Johnson, and T.D. de la Rubia, private communication.

[6] M.D. Giles et al., TED SIMS data from Intel Corporation.
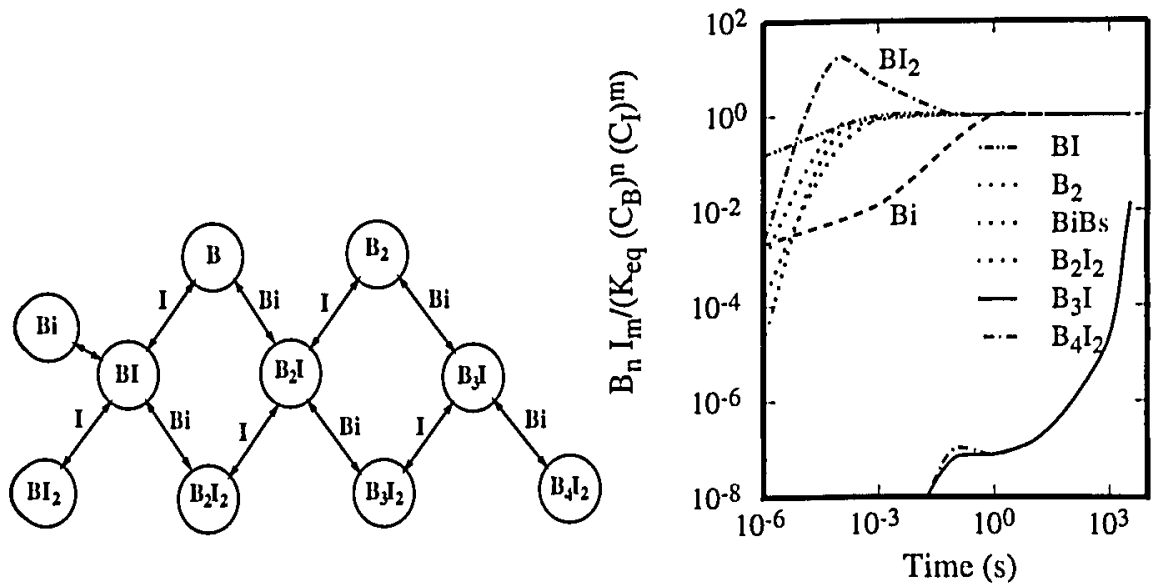

Fig. 1: Cluster reactions (a) considered in the full model as given by fundamental physical calculations [5]. Simulations (b) using the full cluster model of normalized cluster concentrations (relative to their equilibrium value) versus time for an $800^{\circ} \mathrm{C}$ anneal. 

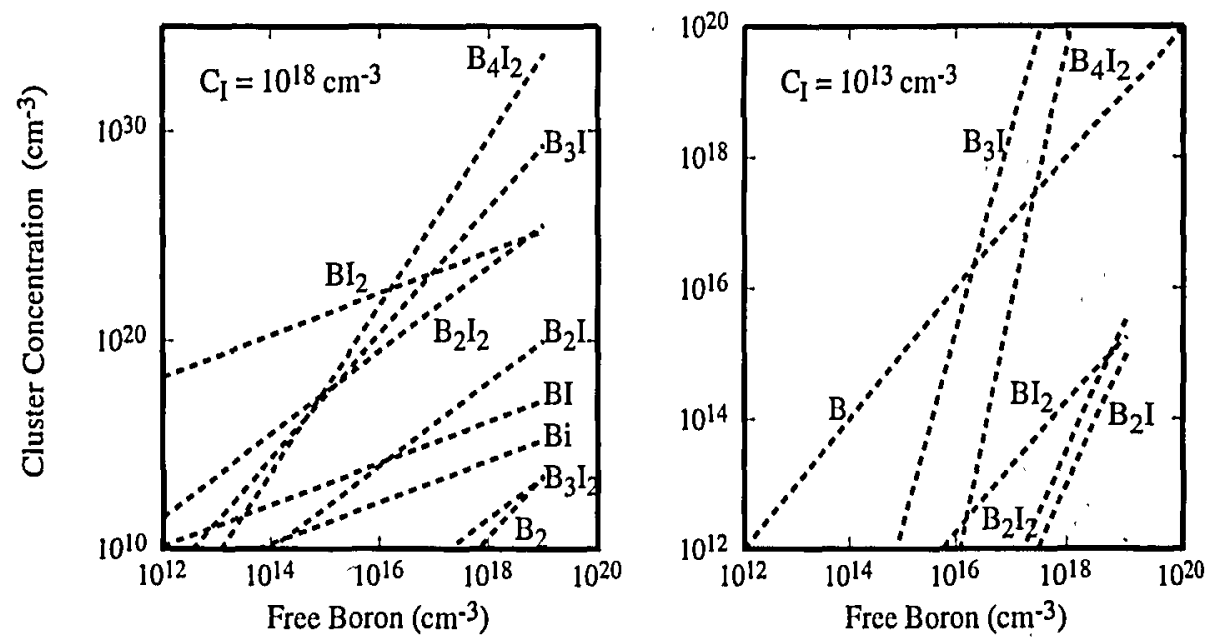

Fig. 2: Equilibrium cluster concentrations versus free boron concentration at $800^{\circ} \mathrm{C}$ for a free interstitial concentration of (a) $10^{18} \mathrm{~cm}^{-3}$ characteristic of very early stages of TED, and (b) $10^{13} \mathrm{~cm}^{-3}\left(C_{\mathrm{I}} / C_{\mathrm{I}}^{*} \sim 10^{4}\right)$ typical of TED conditions in the presence of $\{311\}$ defects. Initially, $\mathrm{BI}_{2}$ is the primary cluster and helps immobilize the boron as noted by Pelaz et al. [4], while $B_{3} I$ is the primary cluster during most of the anneal.
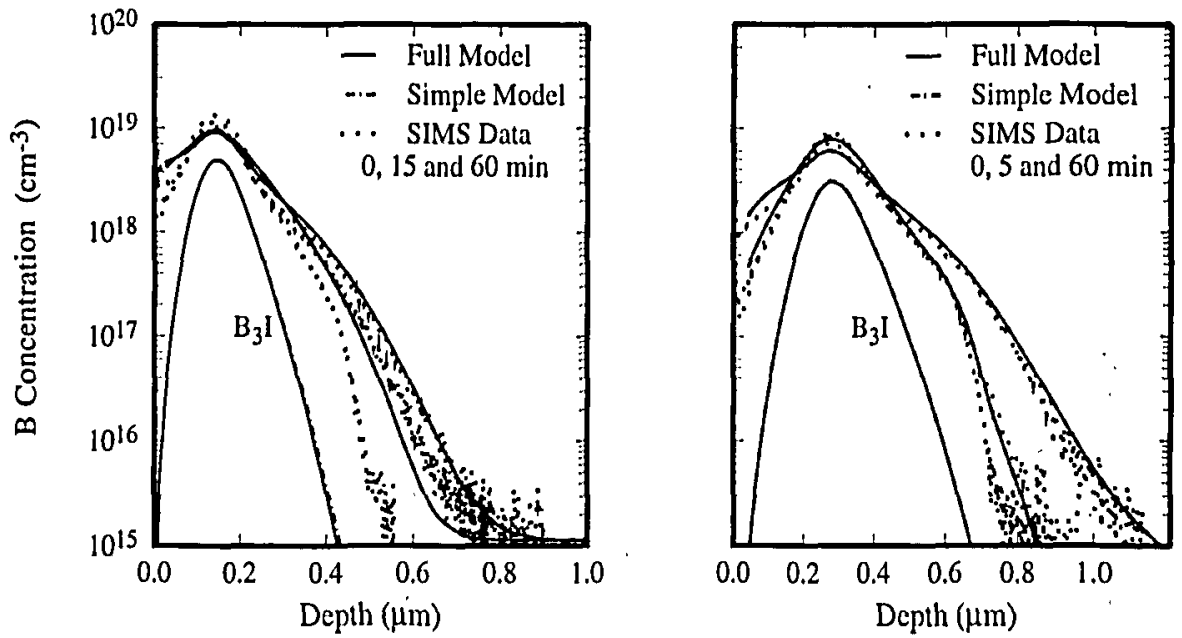

Fig. 3: Comparison of full model with the simplified model for (a) $40 \mathrm{keV}$ and (b) $80 \mathrm{keV}, 2 \times 10^{14} \mathrm{~cm}^{-2} \mathrm{~B}$ implants annealed at $800^{\circ} \mathrm{C}$ for various times. Also shown for comparison are SIMS data from Intel [0]. Note that the full model and simple model show indistinguishable final profiles. The $\mathrm{B}_{3} I$ concentrations for the two models (shown after a 1 , h anneal) are also nearly identical. 